\title{
False Vacuum Decay in Jordan-Brans-Dicke Cosmologies
}

Richard Holman, ${ }^{1}$ Edward W. Kolb, ${ }^{2}$ Sharon L. Vadas, ${ }^{2}$ Yun Wang, ${ }^{1}$ and Erick J. Weinberg ${ }^{3}$

${ }^{1}$ Physics Department, Carnegie Mellon Ciniversity, Pittsburgh PA 15213

\author{
${ }^{2}$ NASA/Fermilab Astrophysics Center \\ Fermi National Accelerator Laboratory, Batavia, IL 60510 \\ and \\ Department of Astronomy and Astrophysics and Enrico Fermi Institute \\ The University of Chicago, Chicago, IL 60637 \\ ${ }^{3}$ Physics Department, Columbia University, New York, .VY 10027
}

\begin{abstract}
We examine the bubble nucleation rate in a first-order phase transition taking place in a background Jordan-Brans-Dicke cosmology. We compute the leading order terms in the nucleation rate when the Jordan-Brans-Dicke feld is large (i.e., late times) by means of a Weyl rescaling of the fields in the theory. We find that despite the fact that the Jordan-Brans-Dicke field (hence the effective gravitational constant) has a time dependence in the false vacuum, at late times the nucleation rate is time independent.
\end{abstract}


Interest in theories with variable gravitational constant has been sekindied acen

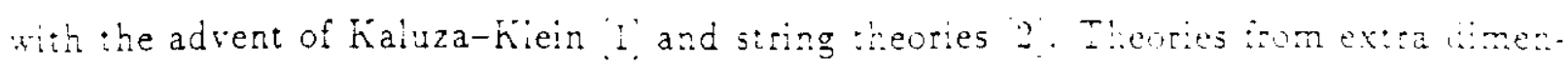
sions typically have a scalar feid. usually cailed the ciiaton. coupled to the Ricci scaia: in a way reminiscent of Jordan-Brans-Dicke (JBD) theories 3. The JBD theories have often been used as toy models to gain insight into more complicated theories. One of the most obvious questions to ask is how these theories differ from standard Einstein gravity in their cosmological evolution. We expect that the cosmological behaviour of such theories at early times will in general be quite different from the standard cosmology 1.

Our main interest here involves the nucleation of bubbles of true vacuum in first-order phase transitions. Steinhardt and La 5 ! have recently used a JBD theory coupled to a scalar field theory admitting a false vacuum to try to save the old inflation model of Guth 6 i. Their model, dubbed extended inflation, represents an interesting new addition to the existing panoply of infiationary models 4 ] (despite the fact that it too may have some problems $:[, 8]$ ), since the fine-tuning problems usually found in new inflation models 4,9 ! can be eliminated.

In this letter we examine the effects upon the bubble nucleation rate of a timedependent background JBD field. Although we only consider JBD theories, our results can be triviaily extended to other models with varying gravitational constants, e.g, induced gravity models where the Ginzburg-Landau scalar field plays the role of the JBD field [10], or Kaluza-Klein and string theory cosmologies where the dilaton field plays the analogous role [11]. While one would expect that a complete calculation of the effects of the JBD field and the metric on the nucleation rate would yield a time dependent nucleation rate (due to the time dependence of the JBD field in the false vacuum), we find that at late times (or equivalently, for large values of the JBD field), the rate becomes approximately time independent. Thus, at least in this regime, the calculations of Refs. 


\section{8 .12 .23 are appiicable.}

Let us start with the action used by Steinharct and La:

$$
\Sigma_{g} g, \Phi, \sigma .=\int d^{4} x \sqrt{-g}\left[-\Phi R-\dot{q}^{\mu \nu} \frac{\partial_{\mu} \Phi \partial_{\nu} \Phi}{\Phi}-\frac{1}{2} g^{\mu \nu} \partial_{\mu} \sigma \partial_{\nu} \sigma-V(\sigma)\right] .
$$

Here $\sigma$ is the scalar matter feld that plays the role of the "inflaton" in extended inflation models. We assume that $\sigma$ has a potential $V^{\prime}(\sigma)$ with a metastable minimum at $\sigma=\sigma_{\mathrm{Fv}}$ with $V\left(\sigma=\sigma_{\mathrm{FV}}\right) \equiv \rho_{V} \neq 0$. and a true ground state at $\sigma=\sigma_{0}$ with $V\left(\sigma_{0}\right)=0 . \Phi$ is the JBD field (with dimension mass ${ }^{2}$ ), and $g_{\mu \nu}$ is the metric tensor. Any time dependence in the JBD field will be manifest as a time dependence of the coefficient of $R$, which serves as an "effective" gravitational constant: $\Phi=1 / 16 \pi G_{\mathrm{EFF}}$. The theory is characterized by a dimensionless parameter $\omega$; in the limit $\omega \rightarrow \infty$ the theory is identical to general relativity. Present experimental limits on $\omega$ require $\omega \gtrsim 500$ [14].

We will be interested in cosmological solutions to the theory where the metric tensor and the fields depend only upon time, and the line element takes the Robertson-Walker form $d s^{2}=d t^{2}-a^{2}(t) d l^{2}$, where $a(t)$ is the cosmological scale factor and $d l^{2}$ is the spatial metric

$$
d l^{2}=\frac{d r^{2}}{1-k r^{2}}+r^{2} d \theta^{2}+r^{2} \sin ^{2} \theta d \phi^{2},
$$

where $k=1,0$, or -1 for a closed, spatially flat, or open Universe.

With the assumptions of a Robertson-Walker metric, $\sigma=$ constant, and $\Phi=\Phi(t)$, the field equations become

$$
\begin{aligned}
\left(\frac{\dot{a}}{a}\right)^{2}+\frac{k}{a^{2}} & =\frac{\rho_{V}}{6 \Phi}+\frac{\omega}{6}\left(\frac{\dot{\Phi}}{\Phi}\right)^{2}-\frac{\dot{a}}{a} \frac{\dot{\Phi}}{\Phi} \\
\ddot{\Phi}+3 \frac{\dot{a}}{a} \dot{\Phi} & =\frac{2 \rho_{V}}{3+2 \omega},
\end{aligned}
$$

where a dot denotes $d / d t$ and $\rho_{V}=-p_{V}=V\left(\sigma=\sigma_{\mathrm{FV}}\right)$ is the (constant) vacuum energy. We will assume that the curvature term in Eq. (3) can be neglected and set $k=0$. The 
resuiting equations have a solution in which the scale facior a tincreases as a iarge; power 0 ime, and the JBD fieid aiso increases with time 15:

$$
\begin{aligned}
& \Phi(t)=\Phi(0)(i-B t)^{2} \\
& a(t)=a(0)(1-B t)^{\omega-1 / 2}
\end{aligned}
$$

where

$$
\begin{aligned}
B & \equiv \frac{1}{\alpha}\left[\frac{\rho_{V}}{6 \Phi(0)}\right]^{1 / 2} \\
\alpha^{2} & \equiv(3-2 \omega)(5-6 \omega) / 12 .
\end{aligned}
$$

(This is not the most general $k=0$ solution, although a large class of solutions approach this form at large times $7 !$ )

So long as $\sigma=$ constant, $a(t)$ and $\Phi(t)$ will increase in time. If there is a metastable minimum at $\sigma=\sigma_{\mathrm{FV}}$, eventually bubbles of true vacuum with $\sigma \approx \sigma_{0}$ will be nucleated. It is this nucleation rate which we wish to study.

The field theoretic calculation of the decay of the false vacuum in flat space-time was essentially solved by Coleman and Callan and Coleman [16]. Incorporation of the gravitational effects upon the nucleation rate was studied by Coleman and DeLuccia [17!. However, the latter analysis is not directly applicable here because of the nonstandard gravitational action whose effective coupling constant changes with time. In order to sidestep these difficulties, let us recast the theory in a form with a time-independent gravitational coupling constant. To do this, we perform a Weyl rescaling [18] to remove the coupling of $\Phi$ to $R$. In more detail, we define new fields $\bar{g}$ and $\psi$ by

$$
\begin{aligned}
g_{\mu \nu} & =\Omega^{2}(x) \bar{g}_{\mu \nu} \\
\psi & =\psi_{0} \ln \left[\Phi(3+2 \omega) / \psi_{0}^{2}\right]
\end{aligned}
$$

where $\psi_{0}^{2}=(3+2 \omega) / 16 \pi G_{N}$ with $G_{N}$ the presently observed value of Newton's constant. 
Choosing the conformai factor $\Omega(x)$ to satisfy $\Omega^{2} \Phi=1: 16 \pi G_{V}$ changes the action to

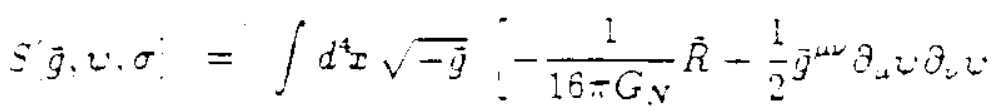

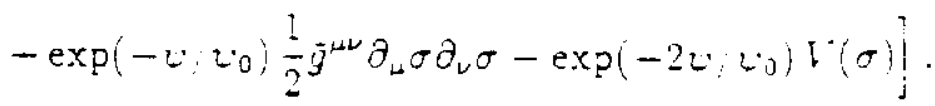

Thus, we now have a system consisting of standard Einstein gravity (with constant gravitational constant), a minimally coupled field $\psi$, and the inflaton field $\sigma$ now coupled to $\psi$. A similar approach was used by Gross and Perry in! in their analysis of monopoles in Kaluza-Klein theories.

How can a theory with a variable $G$ become a theory with a constant $G$ ? The simple answer is that there are two mass scaies, a gravitational mass scale and a mass scale in $V(\sigma)$, but the only relevant observable is the ratio of the two mass scales. In the theory as written as in Eq. (1), the $\sigma$ mass is constant in time while the gravitational mass changes, while in the theory as expressed in Eq. ( 7$)$, the gravitational mass is constant but the $\sigma$ mass varies. Of course, in both pictures the ratio of the gravitational mass to the $\sigma$ mass evolves in time in the same way, and one may choose to work with whichever picture is more convenient.

Again making the assumption that the metric is of the Robertson-Walker form, $\sigma=$ constant, and $\psi$ only depends upon time, the equations of motion are

$$
\begin{aligned}
\left(\frac{\bar{a}^{\prime}}{\bar{a}}\right)^{2}+\frac{k}{\bar{a}^{2}}=\frac{8 \pi G_{N}}{3}\left[\frac{1}{2} \psi^{\prime 2}+\exp \left(-2 \psi / \psi_{0}\right) \rho_{V}\right] \\
\psi^{\prime \prime}+3 \frac{\bar{a}^{\prime}}{\bar{a}} \psi^{\prime}=\frac{2}{\psi_{0}} \exp \left(-2 \psi / \psi_{0}\right) \rho_{V} .
\end{aligned}
$$

In Eq. (8) we have denoted the time and scale factor in the rescaled system of Eq. (7) as $\bar{t}$ and $\bar{a}(\bar{t})$, respectively, and prime denotes $d / d \bar{t}$. The $k=0$ equations of motion again have a power law solution:

$$
\begin{aligned}
& \bar{a}(\bar{t})=\bar{a}(0)(1+C \vec{t})^{3 / 4+\omega / 2} \\
& \psi(\bar{t})=\psi(0)+\psi_{0} \ln (1+C \bar{t}),
\end{aligned}
$$


where here,

$$
C=\frac{1}{\alpha+(0)}{\frac{\rho \vartheta}{2+\pi G_{Y j}}}^{1.2}=2: 6-G_{Y}+(0)^{-12} B .
$$

At this point we would like to see how the two solutions are related to one another. We first note that the conformal factor in our Weyl rescaling is given by

$$
\Omega(t)=16 \pi G_{N} \Phi(t)^{\cdot-1 / 2}=\frac{C}{2 B}(1-B t)^{-1}
$$

Since the two line elements $d \bar{s}^{2}=d \bar{t}^{2}-\vec{a}^{2}(\bar{t}) d l^{2}$ and $d s^{2}=d t^{2}-a^{2}(t) d l^{2}$ are related by $d s / d \bar{s}=\Omega$. we must have $d \bar{l}=d l$ and

$$
\frac{d t}{d \bar{t}}=\frac{a(t)}{\bar{a}(\bar{t})}=\Omega(t)
$$

Integrating the $d \bar{t} / d t$ equation and fixing the constant of integration so that the $\bar{a}(\bar{t}) / a(t)$ equation is satisfied, we obtain

$$
(1+C \vec{t})=(1+B t)^{2} \text {. }
$$

Having completed these preliminaries, let us turn to the the bubble nucleation rate. First, we note that the conformal relation between the original and the rescaled theories fixes the relationship between the nucleation rates in the two theories. In the latter theory the nucleation probability per physical volume per time is

$$
\bar{\lambda}(\bar{t}) \equiv \frac{d P(\bar{t})}{d \bar{V}(\bar{t}) d \bar{t}}
$$

where $P(\bar{t})$ is the probability of bubble nucleation and $\bar{V}(\bar{t})$ is the physical volume measured in the rescaled system at time $\bar{t}$. The equivalent rate in the original theory is then

$$
\lambda(t) \equiv \frac{d P(t)}{d V(t) d t}=\left(\frac{\bar{a}(\bar{t})}{a(t)}\right)^{3} \frac{d \bar{t}}{d t} \bar{\lambda}(\bar{t})=\Omega^{-4}(t) \bar{\lambda}(\bar{t})
$$


where we have used Eqs. $1:)$ and $(23)$ and he iact that $P(t)=P \bar{t}(t)$.

In the JBD theory there are two effects wich mignt cause the bujote nucieation rate to devate from its Aat-space vaiue. Itse Sist is the gravitational couping, while the second is the time dependence of the JBD field. In the rescaled formulation of the theory the gravitational interaction has the standard form, and so we might expect direct gravitational effects to be similar to those in a standard theory. In order to isolate any time dependence coming directly from the JBD field, let us consider the theory of Eq. $(i)$ in the $G_{N} \rightarrow 0$ limit. In this limit the first, gravitational, term in the action should certainly be omitted. What is perhaps less obvious is that the second term, containing the kinetic energy of the JBD field, should also be neglected. To make this a bit clearer, consider replacing $\psi(x)$ by $\dot{\psi}(\boldsymbol{x}) \equiv \psi(\boldsymbol{x}) / \psi_{0}$. The JBD kinetic energy term now has the form $\frac{1}{2} \psi_{0}^{2} \bar{g}^{\mu \nu} \partial_{\mu} \bar{\psi} \partial_{\nu} \bar{\psi}$. Because $\psi_{0}$ is proportional to $G_{N}^{-1 / 2}$, this term scales in precisely the same way as the Ricci term as $G_{N} \rightarrow 0$.

We are thus left with the last two terms of Eq. ( $T$ ). Only the matter field $\sigma$ remains as a dynamical quantity. Without their time derivative terms, the metric and the JBD field now enter essentially as nondynamical, externally specified quantities. Since gravitational effects can only be small if the bubble size is much less than the curvature of space, we take the metric to be flat, and write the action of this truncated theory as

$$
\hat{S}[\sigma]=\int d^{4} x\left[\frac{1}{2} b\left(\partial_{\mu} \sigma\right)^{2}+b^{2} V(\sigma)\right]
$$

where we have defined $b \equiv \exp \left(-\psi / \psi_{0}\right)$. We now wish to calculate the nucleation rate in this truncated theory, or, more precisely, to determine its dependence on the value of $b$. With the gravitational interaction no longer present, we can use the methods of Ref. [16], although with some modifications necessitated by the nonstandard form of the kinetic energy term. The first step is to find the "bounce" $\bar{\sigma}(x)$; i.e., the minimum action solution of the Euclidean equations of motion containing a true vacuum region where 
$\sigma \approx \sigma_{0}$ but with $\sigma$ approaching its false vacuum value at infinity. The bubbie sucteation ate per unit volume is ihen given $b_{y} \bar{\lambda}=A \exp \left(-\Sigma_{E}\right)$, where $\Sigma_{E}$ is the Eucidean action of the bounce wille the prefactor $A$ is given by

$$
A=\frac{\operatorname{det}^{\prime} i S_{E}^{\prime \prime}(\bar{\sigma}) !}{\operatorname{det} S_{E}^{\prime \prime}\left(\sigma_{F V}\right)} \prod_{\omega}^{-1 / 2}\left(\frac{C_{\omega}}{2 \pi}\right)^{1.2} .
$$

Here, the prime denotes that the functional determinant is to be evaluated in the subspace orthogonal to the four transiational zero modes. The $C_{\mu}$ are normalization factors for these zero modes, defined so that the properly normalized modes are $C_{\mu}^{-1 / 2} \partial_{\mu} \vec{\sigma}, \mu=1 \ldots t$. so that

$$
C_{\mu}=\int d^{4} x\left(\partial_{\mu} \bar{\sigma}\right)^{2}
$$

with no sum over $\mu$ implied. (With an $O(4)$-symmetric bounce, the $C_{\mu}$ are all equal and can be simply related to the bounce action; we will not need to use this fact.)

By a simple rescaling of coordinates, one finds that the bounce solution has the form $\sigma(x)=\dot{\sigma}(\sqrt{b} x)$, where $\dot{\sigma}(y)$ is the bounce solution when $b=1$. By substituting this into the action and then rescaling the integration variable, we see that $S_{E}$ is the same as for the $b=1$ case; all of the $b$ dependence lies in the prefactor. Performing the functional variation of the Euclidean action gives

$$
A_{D E T} \equiv\left|\frac{\operatorname{det}^{\prime}\left[S_{E}^{\prime \prime}(\bar{\sigma})\right]}{\operatorname{det}\left[S_{E}^{\prime \prime}\left(\sigma_{F V}\right)\right]}\right|^{-1 / 2}=\left|\frac{\operatorname{det}^{\prime}\left[-b \partial^{2}+b^{2} V^{\prime \prime}(\vec{\sigma})\right]}{\operatorname{det}\left[-b \partial^{2}+b^{2} V^{\prime \prime}\left(\sigma_{\mathrm{FV}}\right)\right]}\right|^{-1 / 2} \text {. }
$$

To determine the $b$ dependence of the determinants, we note that if $\Psi_{\lambda}(x)$ is an eigenfunction of the operator $-\partial^{2}+V^{\prime \prime}(\hat{\sigma})$ with eigenvalue $\lambda$, then $\Psi_{\lambda}(\sqrt{b} x)$ will be an eigenfunction of the operator $-b \partial^{2}+b^{2} V^{\prime \prime}(\bar{\sigma})$ with eigenvalue $b^{2} \lambda$; the same relationship holds for the eigenvalues associated with the false vacuum configuration. Now if we note that the primed determinant has four fewer eigenvalues than the unprimed one, we see that $A_{D E T}$ contains a factor of $\left[\left(b^{2}\right)^{-4}\right]^{-1 / 2}=b^{4}$; i.e., $A_{\mathrm{DET}}=b^{4} \hat{A}_{\mathrm{DET}}$, where $\dot{A}_{\mathrm{DET}}$ is the determinant ratio $b=1$. Finally, the $b$ dependence of the $C_{\mu}$ can be obtained by substituting 
our bounce soiution into Eq. (18) and rescaling the integration variable. The resut is that $C_{\mu}=b^{-1} \dot{C}_{\mu}$, where again the hat indicates the $b=i$ value. The pretacior $A$ inus has a net factor of $b^{2}$, so that $\bar{\lambda}(\bar{t})=b^{2} \lambda_{0}$, where $\lambda_{0}$ is the nucleation rate for a normal scalar field theory with potential $V(\sigma)$. Since $b$ is a function of the time-dependent JBD field, we thus have a time-dependent nucleation rate in the rescaled theory. However, this time dependence disappears if we go back to the original theory. Eqs. (6) and (11), together with the definition of $\psi_{0}$, imply that $b=\Omega^{2}(t)$. Using Eq. (15), we find that the nucleation rate of the original theory is $\lambda(t)=\lambda_{0}$, and is thus time independent in the approximation to which we are working. Indeed, this is just the result one would have obtained by omitting both gravitational and JBD effects from the original action of Eq. (1).

It may be a bit unsettling that the nucleation rate can be either time dependent or time independent, depending on the framework in which one chooses to work, but this is inevitable once we exploit the possibility to make a time-dependent rescaling of spacetime. The ambiguity is eliminated if one asks questions which are phrased entirely in terms of physical lengths. Thus, if we calculate the probability that a bubble will nucleate within a space-time volume equal to the fourth power of the $\sigma$-Compton wavelength, the result will be the same in either formalism and, in the limit we are working, will be independent of time.

Similarly, we should require that both frameworks give the same answer to the question of whether and, if so, when the true vacuum percolates. Some indication of this can be found by considering the dimensionless parameter $\epsilon \equiv \lambda / H^{4}(t)$ where $H(t) \equiv \dot{a}(t) / a(t)$ $[7,8,12]$. In a de Sitter universe described by general relativity, percolation occurs if this parameter is larger than a critical value, $\epsilon_{C R}$, lying in the range $1.1 \times 10^{-6} \lesssim \epsilon_{C R} \lesssim 0.24$ [12]; the same is expected to be true in the large-w limit of JBD theory. To compare our two theories, we calculate $\bar{\epsilon}(\bar{t}) \equiv \bar{\lambda} / \bar{H}(\bar{t})$. Using our formulae above, and making use of 
the relation between $\bar{t}$ and $t$, we find that

$$
\left.\bar{t} \cdot \bar{t})=\left(\frac{2 \omega-\vdots}{2 \omega-3}\right)^{t} \epsilon t\right)
$$

so that the two theories agree in the limit of large $s$.

Let us comment on the vaidity of our approximations. We have worked in the iimit $G_{N} \rightarrow 0$. Although we have not yet calculated the first order corrections to this limit, we can use the results of bubble nucleation calculations in standard gravity as a guide. In those calculations, the terms of order $G_{. V}$ are small provided that the mass scales in the matter part of the action are much less than the Planck mass, and that the bubble size is small compared to the curvature of space. By analogy, we expect our approximation to be reliable when the effective Planck mass induced by the JBD field is much greater than the mass scales associated with the $\sigma$ field. Since $\Phi$ increases with time, our approximation should be best in the large-t limit.

We should also comment on our assumption that $\psi$ could be treated as constant during the bubble nucleation process. This would imply that $\Phi$ was also constant. However, we have explicitly used the fact that both $a$ and $\Phi$ were time dependent in order to derive our result for $\bar{\lambda}$. The resolution of these seemingly contradictory assumptions is that the (imaginary-time) bounce configuration used in computing the tunnelling action is distinct from the (real-time) backround metric and JBD field configuration governing the evolution of the Universe. It is this latter configuration that remains time dependent. A similar situation occurs in old inflation nucleation rate calculations, in which the metric is frozen out of the bounce solution, yet the real-time Universe is still expanding exponentially.

To conclude, we see that at least at large times, the influence of the time dependence of the JBD field does not give rise to any time dependence of the nucleation rate. It would be of great interest to understand how to calculate the full consequences of the 
JBD feld on this rate. This is being done by us $20^{\circ}$, but there are some questions of principle, such as how to specify boundary conditions for the JBD heid in the jounce configuration. We also note that if one chooses to work in the rescaled system where gravity is normal, microphysics is abnormal, and one must take care in calculation of bubble nucleation.

Gravitational effects on vacuum decay in JBD theories have also been considered by Accetta and Romanelli 21 .

\section{Acknowledgements}

We would like to thank F. Accetta for helpful comments. R.H. and Y.W. Were supported in part by DOE grant \# DE-A C02-76ER03066, E.W.K. and S.L.V. were supported in part by the DOE and NASA (grant \# NAGW-1340), and E.J.W. was supported in part by DOE contract \# DE-AC02-76ER02271.

\section{References}

1. T. Kaluza, Preus. Acad. Wiss. K1 (1921) 966; O. Klein, Zeit. Phys. 37 (1926) 895 and Nature 118 (1926) 516; M. J. Duff, B. E. W. Nilsson, and C. N. Pope, Phys. Rep. 130 (1986) 1.

2. M. B. Green, J. H. Schwartz, and E. Witten, Superstring Theory (Cambridge Univ. Press, Cambridge, 1987).

3. P. Jordan, Zeit. Phys. 157 (1959) 112; C. Brans and C. H. Dicke, Phys. Rev. 124 (1961) 925.

4. E. W. Kolb and M. S. Turner, The Early Universe (Addison-Wesley, Redwood City, Ca., 1989).

5. D. La and P. J. Steinhardt, Phys. Rev. Lett. 62 (1989) 376. 
6. A. H. Guth, Phys. Rer, D 23 (1931) 347.

i. E. J. Weinberg. Fhys. Rer. D 40 (1999) 3950.

3. D. La. P. J. Steinhardt, and E. Bertschinger. Phys. Lett. 231 B (1989) 231.

9. A. D. Linde, Phys. Lett. 108B (1982) 389: A. Albrecht and P. J. Steinhardt. Fhys. Rev. Lett. 48 (1982) 1220.

10. F. S. Accetta and J. J. Tiester, Phys. Rev. D 39 (1989) 2954.

11. See, e.g., Yu. A. Kubyshin, V. A. Rubakov, and I. I. Tkachev, International Juumal of Modern Physics A 4 (1989) 1409.

12. A. H. Guth and E. J. Weinberg, Nucl. Phys. B212 (1982) 321.

13. D. La and P. J. Steinhardt, Phys. Lett. 220B (1989) 375.

14. R. D. Reasenberg, et al., Astrophys. J. 234 (1979) L219.

15. See, e.g., C. Mathiazhagen and V. B. Johri, Class. Quantum Grav. 1 (1984) L29.

16. S. Coleman, Phys. Rev. D 15 (1977) 2929; C. G. Callan and S. Coleman, Phys Rev. $D 16(197 \imath) 1762$.

17. S. Coleman and F. De Luccia, Phys. Rev. D 21 (1980) 3305.

18. N. D. Birrell and P. C. W. Davies, Quantum Fields in Curved Space (Cambridge Univ. Press, Cambridge, 1982).

19. D. J. Gross and M. J. Perry, Nucl. Phys. B228 (1983) 29.

20. R. Holman, E. W. Kolb, S. L. Vadas, and Y. Wang, in preparation (1989).

21. F. S. Accetta and P. Romanelli, Yale University preprint YCTP P18-89. 